\title{
AN EXTENDED INHOMOGENEOUS MINIMUM
}

\author{
E. S. BARNES
}

(Received 5 September 1975)

\begin{abstract}
A new arithmetic invariant $E(f)$ is defined for integral binary quadratic forms $f$. It has the property that, denoting by $f_{m}$ the norm-form of a quadratic number field $Q(V m), E\left(f_{m}\right)<1$ if and only if $Q(\vee m)$ has class number one.
\end{abstract}

\section{1}

The inhomogeneous minimum of a form has proved to be an important concept in the study of algebraic number fields with a Euclidean algorithm. I present here a generalization of this concept, for integral binary quadratic forms, which bears a similar relation to the question of unique factorization in quadratic fields.

Let $f(x, y)=a x^{2}+b x y+c y^{2}$ be a binary quadratic form with real coefficients and discriminant $D=b^{2}-4 a c$. The inhomogeneous minimum $M(f)$ of $f$ may be defined thus: for real $x_{0}, y_{0}$, and writing $x_{0}=\left(x_{0}, y_{0}\right)$, set

$$
M\left(f ; x_{0}\right)=\inf _{(x, y) \in \Gamma}\left|f\left(x+x_{0}, y+y_{0}\right)\right|,
$$

where $\Gamma$ denotes the integral lattice in the plane; then

$$
M(f)=\sup _{x_{0}} M\left(f ; x_{0}\right) .
$$

Suppose from now on that $f$ is a primitive integral form, and let $\mathscr{S}=\mathscr{S}(f)$ be the set of linear transformations of the plane with matrix of the form

$$
T=\left(\begin{array}{cc}
t & -c u \\
a u & t+b u
\end{array}\right) \quad t, u \text { integral. }
$$

It is easily seen that, under composition of transformations, $\mathscr{S}$ is a semigroup with identity $I$. (This follows most easily from the fact that an integral $T$ belongs to $\mathscr{P}$ if and only if $T$ transforms $f$ into $(\operatorname{det} T) f$.) 
For $\boldsymbol{x}_{0} \notin \Gamma$, define

$$
E\left(f ; x_{0}\right)=\inf _{\substack{T \in \mathcal{S} \\ T x_{0} \notin \Gamma}} M\left(f ; T x_{0}\right)
$$

and

$$
E(f)=\sup _{x_{0} \notin \Gamma} E\left(f ; x_{0}\right) .
$$

We call $F(f)$ the 'extended inhomogeneous minimum' of $f$. Trivially, since $I \in \mathscr{S}$,

$$
E(f) \leqq M(f)
$$

for all $f$. Note also that since the transformations $T$ of $\mathscr{S}$ are integral,

$$
E\left(f ; x_{1}\right)=E\left(f ; x_{0}\right) \quad \text { if } \quad x_{1} \equiv x_{0}(\bmod \Gamma) .
$$

We show first that, like $M(f), E(f)$ is an arithmetical invariant.

LEMMA 1.1. If $g$ is equivalent to $f$ (under integral unimodular tranformation) then $E(g)=E(f)$.

Proof. Suppose that $g(x)=f(U x)$ where $U$ is integral unimodular, and so $U \Gamma=\Gamma$. Then, for all $x_{0}$, it is easily seen that

$$
M\left(f ; \boldsymbol{x}_{0}\right)=M\left(g ; U^{-1} \boldsymbol{x}_{0}\right) .
$$

Hence

$$
M\left(f ; T x_{0}\right)=M\left(g ; U^{-1} T U\left(U^{-1} x_{0}\right)\right)
$$

The required result will follow at once when we show that

$$
\mathscr{P}(g)=U^{-1} \mathscr{P}(f) U
$$

and for this it suffices, by symmetry, to show that

$$
U^{-1} \mathscr{P}(f) U \subseteq \mathscr{P}(g) .
$$

A straightforward calculation shows that, with

$$
f(x, y)=a x^{2}+b x y+c y^{2}, \quad g(x, y)=a^{\prime} x^{2}+b^{\prime} x y+c^{\prime} y^{2},
$$

if $\operatorname{det} U=1$ and $T \in \mathscr{S}(f)$ is given by (1.3),

$$
U^{-1} T U=\left(\begin{array}{cc}
t+\frac{1}{2}\left(b-b^{\prime}\right) u & -c^{\prime} u \\
a^{\prime} u & t+\frac{1}{2}\left(b+b^{\prime}\right) u
\end{array}\right) \in \mathscr{P}(g) .
$$

A similar calculation shows that $U^{-1} T U \in \mathscr{S}(g)$ also if $\operatorname{det} U=-1$, so (1.7) is proved. 
To establish the connection with unique factorization in quadratic fields, let $F=Q(\vee m)$ where $m$ is square-free and not 0 or 1 . Set

$$
f_{m}(x, y)=\left\{\begin{array}{lll}
x^{2}-m y^{2} & \text { if } \quad m \equiv 2 \text { or } 3(\bmod 4) \\
x^{2}+x y+\frac{1}{4}(1-m) y^{2} & \text { if } \quad m \equiv 1(\bmod 4) .
\end{array}\right.
$$

THEOREM 1. $Q(\sqrt{ } m)$ has class number 1 if and only if $E\left(f_{m}\right)<1$.

We first need

Lemma 1.2. If $\boldsymbol{x}_{0}$ is not a rational point, then $E\left(f ; \boldsymbol{x}_{0}\right)=0$.

Proof. Given any $\varepsilon, 0<\varepsilon<1$, we may, by Minkowski's theorem on linear forms, choose integers $x, y, t, u$ not all zero so that

$$
\left|x+t x_{0}-c u y_{0}\right|<\varepsilon
$$

and

$$
\left|y+a u x_{0}+(t+b u) y_{0}\right|<\varepsilon .
$$

Since $\varepsilon<1, t, u \neq 0,0$; hence if $T$ is defined by (1.3), $T \in \mathscr{P}(f)$ and $T \neq 0$ and so, since $x_{0}$ is not rational, $T x_{0} \notin \Gamma$. Hence

$$
E\left(f ; x_{0}\right) \leqq\left|f\left(x+T x_{0}\right)\right|<\varepsilon^{2}(|a|+|b|+|c|) .
$$

Since $\varepsilon$ is arbitrary, we have $E\left(f ; \boldsymbol{x}_{0}\right)=0$.

Proof of Theorem 1. The Dedekind-Hasse criterion states (see for example Pollard 1950):

if $F$ is an algebraic number field and $J$ its ring of integers, then $F$ has class number 1 if and only if, given any non-zero elements $\alpha, \beta$ of $J$ with $\beta \nmid \alpha$, $\exists \gamma, \delta \in J$ satisfying

$$
0<|N(\alpha \gamma+\beta \delta)|<|N \beta|
$$

(where $N$ is the norm in $F / Q$ ). Setting $\rho=\alpha / \beta$, so that $\rho \notin J$, we can write this condition as: given any $\rho \in F-J, \exists \gamma, \delta \in J$ satisfying

$$
0<|N(\gamma \rho+\delta)|<1 \text {. }
$$

Trivially, this inequality cannot be satisfied for any $\delta$ if $\gamma \rho \in J$; while if $\gamma \rho \notin J$, $N(\gamma \rho+\delta) \neq 0$ for all $\delta \in J$. Thus we can finally write the condition as: given any $\rho \in F-J, \exists \gamma, \delta \in J$ with $\gamma \rho \notin J$ satisfying

$$
|N(\gamma \rho+\delta)|<1 \text {. }
$$

Let now $F$ be a quadratic field, so that $F=Q(\sqrt{ } m)$ where $m \neq 0$ or 1 and $m$ is square-free. A basis of $J / Z$ is $\{1, \omega\}$, where 


$$
\omega=\left\{\begin{array}{cll}
\vee m & \text { if } & m \equiv 2 \quad \text { or } 3(\bmod 4) \\
\frac{1}{2}(1+\vee m) & \text { if } & m \equiv 1 \quad(\bmod 4)
\end{array}\right.
$$

and then, for rational $x, y$,

$$
N(x+\omega y)=f_{m}(x, y) .
$$

Writing $\rho=x_{0}+\omega y_{0} \quad\left(x_{0}, y_{0} \in Q\right), \quad \gamma=t+\omega u \quad(t, u \in Z), \quad \delta=x+\omega y$ $(x, y \in Z)$, we can translate the Dedekind-Hasse criterion into: $Q(\mathrm{~V} m)$ has class number 1 if and only if, given any rational $x_{0}=\left(x_{0}, y_{0}\right) \notin \Gamma$, there exists a

(1.9) $T=\left(\begin{array}{cc}t & m u \\ u & t\end{array}\right)$ or $\left(\begin{array}{cc}t & \frac{1}{4}(-1+m) u \\ u & t+u\end{array}\right)$ respectively $(t, u \in Z)$

with $T x_{0} \notin \Gamma$ and an $x=(x, y) \in \Gamma$ satisfying

$$
\left|f_{m}\left(T \boldsymbol{x}_{0}+\boldsymbol{x}\right)\right|<1 \text {. }
$$

Since clearly $T$ has the shape (1.9) if and only if $T \in \mathscr{P}\left(f_{m}\right)$, we see that (1.10) holds precisely when

$$
E\left(f_{m} ; \boldsymbol{x}_{0}\right)<1
$$

For irrational $\boldsymbol{x}_{0}$, Lemma 1.2 shows that this inequality is always satisfied; so Theorem 1 follows immediately.

Although it is trivially true that $E(f) \leqq M(f)$ for all $f$, it appears that $E$ does not satisfy any stronger general inequality than $M$. More precisely, we have

THEOREM 2.1. If $f$ is a primitive integral indefinite quadratic form of discriminant $D>0$, then

$$
E(f)<\frac{1}{4} \vee D
$$

and the constant $\frac{1}{4}$ is best possible.

Proof. (i) A well-known result of Minkowski states that, for indefinite $f$,

$$
M(f) \leqq \frac{1}{4} \vee D,
$$

where equality holds only for forms equivalent to a multiple of

$$
f_{0}(x, y)=x y \text {. }
$$

Now $\mathscr{S}\left(f_{0}\right)$ contains the transformations $\left(\begin{array}{ll}1 & 0 \\ 0 & 0\end{array}\right)$ and $\left(\begin{array}{ll}0 & 0 \\ 0 & 1\end{array}\right)$, from which it is easy to see that $E\left(f_{0}\right)=0$. Hence (2.1) now follows from the fact that $E(f) \leqq M(f)$. 
(ii) Consider, for positive integral $k$, the form

$$
\varphi_{k}(x, y)=x^{2}+2 k x y-y^{2}
$$

with discriminant $D=4\left(k^{2}+1\right)$. Davenport (1946) showed that

$$
M\left(\varphi_{k}\right)=M\left(\varphi_{k} ;\left(\frac{1}{2}, \frac{1}{2}\right)\right)=\frac{1}{2} k .
$$

If now $T \in \mathscr{S}\left(\varphi_{k}\right)$,

$$
T=\left(\begin{array}{cc}
t & u \\
u & t+2 k u
\end{array}\right), \quad t, u \in Z
$$

and so

$$
T\left(\frac{1}{2}, \frac{1}{2}\right)=\left(\frac{1}{2}(t+u), \frac{1}{2}(t+u)+k u\right) \equiv\left(\frac{1}{2}(t+u), \frac{1}{2}(t+u)\right)(\bmod \Gamma) .
$$

Hence if $T\left(\frac{1}{2}, \frac{1}{2}\right) \notin \Gamma$, necessarily $T\left(\frac{1}{2}, \frac{1}{2}\right) \equiv\left(\frac{1}{2}, \frac{1}{2}\right)(\bmod \Gamma)$. It follows that

$$
E\left(\varphi_{k} ;\left(\frac{1}{2}, \frac{1}{2}\right)\right)=M\left(\varphi_{k} ;\left(\frac{1}{2}, \frac{1}{2}\right)\right)=\frac{1}{2} k .
$$

The result of the theorem now follows on noting that $\frac{1}{2} k / V D \rightarrow \frac{1}{4}$ as $k \rightarrow \infty$.

For a simple result in the opposite direction, define

$$
\mu(f)=\inf _{\substack{x \in \Gamma \\ x \neq 0}}|f(x, y)|
$$

(the homogeneous minimum of $f$ ).

THEOREM 2.2. If $f(x, y)$ does not represent zero (for integral $x, y \neq 0,0$ ), then

$$
E(f) \geqq \frac{1}{4} \mu(f) \geqq \frac{1}{4} .
$$

Proof. An element $T$ of $\mathscr{S}(f)$ maps each of the points $\left(\frac{1}{2}, 0\right),\left(0, \frac{1}{2}\right),\left(\frac{1}{2}, \frac{1}{2}\right)$ either into a point of $\Gamma$ or into a point of this set modulo $\Gamma$. It follows that

$$
\begin{aligned}
E(f) & \geqq \min \left\{M\left(f ;\left(\frac{1}{2}, 0\right)\right), M\left(f ;\left(0, \frac{1}{2}\right)\right), M\left(f ;\left(\frac{1}{2}, \frac{1}{2}\right)\right)\right\} \\
& \geqq \frac{1}{4} \mu(f) .
\end{aligned}
$$

It is known that there exists a constant $\kappa>0$ such that, if $f$ is indefinite and does not represent zero, then $M(f)>\kappa \vee D$. It is an open question whether a similar result holds for $E(f)$; if it does, it could immediately be deduced from Theorem 1.1 that there exist only finitely many real quadratic fields with class number one (contrary to a well-known conjecture of Gauss!).

\section{The evaluation of $E\left(f_{m}\right)$}

We indicate here a procedure for calculating $E\left(f_{m}\right)$ for given $m$, and in particular for determining whether or not $E\left(f_{m}\right)<1$. The methods apply with 
obvious modifications to any integral form $f$, and indicate the necessarily close relation between the value of $E(f)$ and the number of classes of forms of given discriminant $D=D(f)$. It is convenient here to restrict attention to forms which do not represent zero.

By Lemma 1.2 , it suffices in evaluating $E(f)$ to consider $E\left(f ; x_{0}\right)$ for rational $x_{0} \notin \Gamma$, say $x_{0}=\left(\frac{r}{q}, \frac{s}{q}\right)$ where $\operatorname{gcd}(r, s, q)=1$ and $q \geqq 2$. Since all integral multiples of the identity belong to $\mathscr{S}(f)$ for all $f$, it follows easily that

$$
E\left(f ;\left(\frac{r}{q^{\prime \prime}}, \frac{s}{q^{\prime}}\right)\right) \leqq E\left(f ;\left(\frac{r}{q}, \frac{s}{q}\right)\right) \text { if } q \mid q^{\prime} .
$$

We may therefore restrict our attention to prime $q$, and define for such $q$

$$
E_{q}(f)=\max _{\substack{x_{0} \& T \\ q x_{0} \in \Gamma}} E\left(f ; x_{0}\right),
$$

whence

$$
E(f)=\max _{q \text { prime }} E_{q}(f) .
$$

LEMMA 3.1. Suppose that $f$ does not properly represent zero modulo $q$. Then

$$
E_{q}(f) \leqq \frac{1}{4} \mu(f) .
$$

Proof. By applying a suitable equivalence transformation, we may assume that

$$
f(x, y)=a x^{2}+b x y+c y^{2} \text { with } \mu(f)=|a| .
$$

Let $x_{0}=\left(\frac{r}{q}, \frac{s}{q}\right), x_{0} \notin \Gamma$. Choose

$$
T=\left(\begin{array}{cc}
a r+b s & c s \\
-a s & a r
\end{array}\right) \in \mathscr{S}(f) .
$$

Then

$$
T x_{0}=\left(\frac{1}{q} f(r, s), 0\right)
$$

where, by hypothesis, $f(r, s) \not \equiv 0(\bmod q)$, so that $T x_{0} \notin \Gamma$. Choosing an integer $x$ with $|x+(1 / q) f(r, s)| \leqq \frac{1}{2}$, we obtain

$$
E\left(f ; \boldsymbol{x}_{0}\right) \leqq M\left(f ; T x_{0}\right) \leqq\left|f\left(x+\frac{1}{q} f(r, s), 0\right)\right| \leqq \frac{1}{4}|a|=\frac{1}{4} \mu(f) .
$$


Since this result holds for all $x_{0}$ with $q x_{0} \in \Gamma$, (3.3) follows.

We now look particularly at the forms $f_{m}$.

LEMMA 3.2. If $q$ is prime and $\frac{1}{4}<\lambda \leqq 1$, then $E_{q}\left(f_{m}\right)<\lambda$ if

(i) $m<0, m \equiv 2$ or $3(\bmod 4)$ and $q^{2}>\frac{4|m|}{3 \lambda^{2}}$;

or (ii) $m<0, m \equiv 1(\bmod 4)$ and $q^{2}>\frac{|m|}{3 \lambda^{2}}$;

or (iii) $m>0, m \equiv 2$ or $3(\bmod 4)$ and $q^{2}>\frac{|m|}{2 \lambda^{2}}$;

or (iv) $m>5, m \equiv 1(\bmod 4)$ and $q^{2}>\frac{|m|}{8 \lambda^{2}}$.

Proof. Since $\mu\left(f_{m}\right)=1$, Lemma 3.1 shows that it suffices to consider only primes $q$ for which $f_{m}$ properly represents zero modulo $q$.

Let $x_{0}=\left(\frac{r}{q}, \frac{s}{q}\right), x_{0} \notin \Gamma$. If $f(r, s) \neq 0(\bmod q)$, the argument of Lemma 3.1 shows that $E_{q}\left(f_{m} ; x_{0}\right) \leqq \frac{1}{4}<\lambda$. We may therefore suppose that

$$
f_{m}(r, s) \equiv 0(\bmod q)
$$

and since $r, s \neq 0,0(\bmod q)$, we see that $s \not \equiv 0(\bmod q)$. Hence there exists an integral $z$ with $r \equiv s z(\bmod q)$ and so

$$
x_{0} \equiv\left(\frac{s z}{q}, \frac{s}{q}\right)(\bmod q), \quad s \not \equiv 0(\bmod q)
$$

where

$$
f_{m}(z, 1) \equiv 0(\bmod q) .
$$

It is easily verified that the set of points (3.4) is permuted by the transformations of $\mathscr{S}\left(f_{m}\right)$; and that, although $z$ is not uniquely defined by (3.5), two different $z$ yield the same value of $M\left(f_{m} ; x_{0}\right)$ for the point (3.4). It thus follows that, if $E_{q}\left(f_{m}\right)>\frac{1}{4}$, then

$$
E_{q}\left(f_{m}\right)=\min _{s \neq 0(\bmod q)} M\left(f_{m} ;\left(\frac{s z}{q}, \frac{s}{q}\right)\right)
$$

$z$ is any integer satisfying (3.5).

Now

$$
\begin{aligned}
f_{m}\left(x^{\prime}+\frac{s z}{q}, y^{\prime}+\frac{s z}{q}\right) & =\frac{1}{q^{2}} f_{m}\left(q x^{\prime}+s z, q y^{\prime}+s\right) \\
& =\frac{1}{q^{2}} f_{m}(q x+z y, y),
\end{aligned}
$$


where

$$
x=x^{\prime}-z y^{\prime}, \quad y=q y^{\prime}+s
$$

so that $\left(x^{\prime}, y^{\prime}\right) \in \Gamma$ iff $(x, y) \in \Gamma$ and $y \equiv s(\bmod q)$. Now

$$
\begin{aligned}
\frac{1}{q} f_{m}(q x+z y, y) & =\left\{\begin{array}{l}
q x^{2}+2 z x y+\frac{1}{q}\left(z^{2}-m\right) y^{2} \\
q x^{2}+(2 z+1) x y+\frac{1}{q}\left(z^{2}+z-\frac{m-1}{4}\right) y^{2} \\
m \equiv 1(\bmod 4)
\end{array}\right. \\
& =f_{m}^{(q)}(x, y),
\end{aligned}
$$

say, where $f_{m}^{(q)}$ is an integral quadratic form of discriminant $D=4 m$ or $m$. It now follows from (3.6) that, if $E_{q}\left(f_{m}\right)>\frac{1}{4}$,

$$
E_{q}\left(f_{m}\right)=\frac{1}{q} \min _{\substack{(x, y) \in \Gamma \\ y \neq 0(\bmod q)}}\left|f_{m}^{(q)}(x, y)\right|
$$

By classical results on the homogeneous minima of quadratic forms, there exist $(x, y) \in \Gamma,(x, y) \neq(0,0)$ satisfying

$$
\left|f_{m}^{(q)}(x, y)\right| \leqq \sqrt{\frac{\mid D\rfloor}{3}} \text { if } m<0
$$

and

$$
\left|f_{m}^{(q)}(x, y)\right| \leqq \sqrt{\frac{D}{8}} \text { if } m>0 \text { and } m \neq 5 \text {. }
$$

Hence, firstly, if $m<0$ and $\frac{1}{3}|D|<\lambda^{2} q^{2}$, we have $(x, y) \in \Gamma-\{0\}$ satisfying

$$
\left|f_{m}^{(q)}(x, y)\right|<\lambda q
$$

since $f_{m}^{(q)}(x, y) \equiv 0(\bmod q)$ if $y \equiv 0\left(\bmod (q)\right.$, and since $f_{m}^{(q)}$ is not a zero form, it follows from (3.8) that

$$
E_{q}\left(f_{m}\right)<\lambda
$$

The results (i) and (ii) of the Lemma follow with $|D|=4|m|$ and $|m|$ respectively.

A similar analysis yields the results (iii) and (iv) in the case $m>0(m \neq 5)$.

When $m<0$, it is possible to obtain somewhat stronger results by using the properties of reduced quadratic forms. Suppose that, in (3.5), we choose $z$ to satisfy 


$$
\left\{\begin{array}{lll}
-\frac{1}{2} q<z \leqq \frac{1}{2} q & \text { when } & m \equiv 2 \text { or } 3(\bmod 4) \\
z=0 \text { if } q=2 & \text { and } & m \equiv 1(\bmod 4) \\
-\frac{1}{2}(q+1)<z \leqq \frac{1}{2}(q-1) & \text { when } & m \equiv 1(\bmod 4) \text { and } q \text { is odd }
\end{array}\right.
$$

Then $f_{m}^{(q)}$ is reduced in the sense of Gauss if also

$$
f_{m}^{(q)}(0,1)=\frac{1}{q} f_{m}(z, 1) \geqq q ;
$$

and it then follows that, since $f_{m}^{(q)}(0,1)$ is the least value assumed by $f_{m}^{(q)}(x, y)$ with $y \neq 0$,

$$
E_{q}\left(f_{m}\right)=\frac{1}{q} f_{m}^{(q)}(0,1)=\frac{1}{q^{2}} f_{m}(z, 1):
$$

If however (3.12) does not hold, we have in any case

$$
E_{q}\left(f_{m}\right) \leqq \frac{1}{q} f_{m}^{(q)}(0,1)=\frac{1}{q^{2}} f_{m}(z, 1) .
$$

Summarizing, we have:

LEMMA 3.3. If $m<0, q$ is prime and $z$ satisfies (3.5) and (3.11), then

(i) $E_{q}\left(f_{m}\right)=\frac{1}{q^{2}} f_{m}(z, 1)$ if $f_{m}(z, 1) \geqq q^{2}$;

(ii) $E_{q}\left(f_{m}\right) \leqq \frac{1}{q^{2}} f_{m}(z, 1)$ if $f_{m}(z, 1)<q^{2}$.

We conclude with some examples of the evaluation of $E\left(f_{m}\right)$.

(1) $E\left(f_{-35}\right)=1$. We have

$$
f_{-35}(x, y)=x^{2}+x y+9 y^{2} .
$$

Since the congruence $f_{-35}(z, 1) \equiv 0(\bmod 2)$ is insoluble, $E_{2}\left(f_{-35}\right)=\frac{1}{4}$. Next

$$
f_{-35}^{(3)}(x, y)=3 x^{2}+x y+3 y^{2}
$$

so, by Lemma 3.3 (this form being reduced), $E_{3}\left(f_{-35}\right)=1$. Finally, by Lemma 3.2 (ii), $E_{q}\left(f_{-35}\right)<1$ if $q^{2}>35 / 3$ and so if $q \geqq 5$. Hence

$$
E\left(f_{-35}\right)=E_{3}\left(f_{-35}\right)=1
$$

(2) $E\left(f_{38}\right)=\frac{1}{2}$. First

$$
f_{38}^{(2)}(x, y)=2 x^{2}-19 y^{2} \quad \text { and } \quad f_{38}^{(2)}(3,1)=-1 \text {, }
$$

so that

$$
E_{2}\left(f_{38}\right)=\frac{1}{2}
$$


Next, the congruence

$$
f_{38}(z, 1)=z^{2}-38 \equiv 0(\bmod q)
$$

is insoluble for $q=3,5$ and 7 , whence

$$
E_{q}\left(f_{38}\right) \leqq \frac{1}{4} \text { for } q=3,5 \text { and } 7 .
$$

Finally, by Lemma 3.2 (iii),

$$
E_{q}\left(f_{38}\right)<\frac{1}{2} \quad \text { if } q^{2}>76
$$

and so for all prime $q>7$. Hence

$$
E\left(f_{38}\right)=E_{2}\left(f_{38}\right)=\frac{1}{2} .
$$

(3) $E\left(f_{42}\right)=\frac{3}{2}$. First, $f_{42}^{(2)}(x, y)=2 x^{2}-21 y^{2}$; congruences $\bmod 8$ give

$$
f_{42}^{(2)}(x, y) \equiv \pm 3(\bmod 8)
$$

for odd $y$; also $f_{42}^{(2)}(3,1)=-3$. Hence

$$
E_{2}\left(f_{42}\right)=\frac{3}{2}
$$

Next

$$
f_{42}^{(3)}(x, y)=3 x^{2}-14 y^{2} \text { and } f_{42}^{(3)}(2,1)=-2
$$

so that

$$
E_{3}\left(f_{42}\right) \leqq \frac{2}{3} .
$$

Finally, $E_{q}\left(f_{42}\right)<1$ if $q^{2}>21$, by Lemma 3.2 (iii), and so for all primes $q>3$. Hence

$$
E\left(f_{42}\right)=E_{2}\left(f_{42}\right)=\frac{3}{2}
$$

(4) $E\left(f_{97}\right)=\frac{1}{2}$. Here

$$
f_{97}(x, y)=x^{2}+x y-24 y^{2}
$$

Hence

$$
f_{97}^{(2)}(x, y)=2 x^{2}+x y-12 y^{2} \quad \text { and } \quad f_{97}^{(2)}(19,-7)=1
$$

and so

$$
E_{2}\left(f_{97}\right)=\frac{1}{2}
$$

Next

$$
f_{97}^{(3)}(x, y)=3 x^{2}+x y-8 y^{2} \quad \text { and } \quad f_{97}^{(3)}(3,2)=1
$$

and so

$$
E_{3}\left(f_{97}\right)=\frac{1}{3}
$$


The congruence

$$
f_{97}(z, 1) \equiv 0(\bmod 5)
$$

is insoluble, whence $E_{5}\left(f_{97}\right) \leqq \frac{1}{4}$. Finally, by Lemma 3.2 (iv), $E_{q}\left(f_{97}\right)<\frac{1}{2}$ if $q^{2}>\frac{97}{2}$ and so for all prime $q \geqq 7$. Hence

$$
E\left(f_{97}\right)=E_{2}\left(f_{97}\right)=\frac{1}{2} .
$$

This example is of interest, since $Q(\sqrt{ } 97)$, while simple, is not Euclidean.

\section{References}

H. Davenport, (1946) 'Non-homogeneous binary quadratic forms', Proc. K. Ned. Akad. van Wet. 49, 815-821.

H. Pollard, (1950) The theory of algebraic numbers (Math. Association of America, New York, 1950 ).

University of Adelaide,

South Australia. 